\title{
Waveform Characterization of Calibration-Pulse Generators for EMI Measuring Receivers
}

\author{
Marco A. Azpúrua \\ Universitat Politècnica de \\ Catalunya \\ Barcelona, Spain \\ email: marco.azpurua@upc.edu
}

\author{
Marc Pous \\ Universitat Politècnica de \\ Catalunya \\ Barcelona, Spain \\ email: marc.pous@upc.edu
}

\author{
Ferran Silva \\ Universitat Politècnica de \\ Catalunya \\ Barcelona, Spain \\ email: ferran.silva@upc.edu
}

\author{
Martin Hudlička \\ Czech Metrology Institute \\ Brno, Czech Republic \\ mhudlicka@cmi.cz
}

\begin{abstract}
This paper presents the waveform characterization of calibration pulse generators intended to evaluate the response to pulses of the weighting detectors in CISPR 16-1-1 measuring receivers. First, the standard requirements of the reference pulses are described, and the pulse generators calibration methods based on waveform measurements are briefly discussed. Then, high-resolution time domain measurements are used for characterizing the waveforms of a commercial calibration-pulse generator in terms of rise/fall time, pulse width, mean voltage of the upper state, repetition frequency, and area. Moreover, the results above are used for estimating the spectral density of the impulses, their corresponding quasi-peak level, the pulses bandwidth, and the breakpoint frequencies. Finally, the measurement uncertainty is estimated for CISPR bands A, B, and C/D. Results are in good agreement with other calibrations performed during an intercomparison exercise and the uncertainty satisfy the target $\pm 0.5 \mathrm{~dB}$ and $1 \%$ given in standards for the impulse area and pulse repetition frequency respectively.
\end{abstract}

Keywords-calibration, electromagnetic interference, measuring receiver, pulse response, waveform measurements

\section{INTRODUCTION}

Measuring receivers are the fundamental instrument in emissions testing. In summary, test receivers must measure the amplitude spectrum of an electromagnetic interference according to the indications given in CISPR 16-1-1 [1]. Among those specifications, there are four baseline requirements that are fundamental for ensuring coherent results in EMI assessments, particularly, the sine wave level accuracy, the selectivity, the voltage standing wave ratio (VSWR) and the absolute and relative response to pulses of the weighting detectors [2], [3].

Therefore, it is mandatory to evaluate those baseline parameters to verify if measuring receivers complies with CISPR 16-1-1. Traceable calibrations are used for demonstrating the measuring receiver performance is within the standard tolerances. In that sense, calibration methods for the sine wave level, VSWR and the frequency selectivity are well-established and only require using generic calibration setups comprising instruments such as RF power meters, power sensors, signal generators, vector network analyzers, and accessories like step attenuators and splitters [4]-[6].

Nonetheless, for calibrating of the pulse response of the weighting detectors, the established method, shown in Fig. 1, requires a (nanosecond) pulse generator capable of delivering a set of pulses with a fixed impulse area and repetition frequency [7]. Clearly, the traceability of the receiver's response to pulses calibration requires the pulse generators to be properly calibrated.
In this regard, the calibration of pulse generators is also discussed in the CISPR 16-1-1. Normative annexes B and C of this standard cover the determination of pulse generator spectrum and a method for measuring the output of nanosecond pulse generators. Here, only a brief description of the methods is given, and technical details are hidden. The measurement uncertainty of the pulse generator characterization is not discussed.

In general, a pulse generator is an instrument capable of generating time-domain rectangular pulses, or a pulsemodulated RF signal. Baseband pulses are typically used for lower frequencies (bands $\mathrm{A} / \mathrm{B}$ ) while pulse-modulated RF tones are more suited for higher frequencies (bands $\mathrm{C} / \mathrm{D}$ and E) because of the risk of receiver damage due to high peak voltages. The base-band pulse generators usually comprise of an energy-storage device (electrostatic, magnetic field) and a switch which discharges a fraction or all of the energy into a load. In the past, the switch was realized using a mechanical mercury relay, nowadays mercury relay is replaced by other mechanical principles or a solid-state semiconductor switch is used. Very often the storage device is a charged coaxial line, whereas the pulse duration is given by the electrical length of the line and the impulse area is given by the charge voltage. The charged transmission line does not allow for very fast rising edges. Therefore, step-recovery diodes are used in conjunction with charging and discharging a transmission lines for achieving faster switching. Pulse shapes other than rectangular are usually achieved by TTL pulse shaping.

Because of the extremely short pulse duration, the subnanosecond rise/fall times, and the high peak voltages, characterizing the waveforms of the calibration pulse generators is not a trivial task. This paper presents how highresolution time domain measurements have been used for accurately determining the principal waveform parameters and, most importantly, for ensuring the fulfillment of impulse area and pulse repetition rate. Additionally, the results above are used for estimating the spectral density of the impulses, their corresponding quasi-peak level, the pulses bandwidth, and the breakpoint frequencies. Finally, the measurement uncertainty is estimated for CISPR bands $A, B$, and $C / D$. respectively.

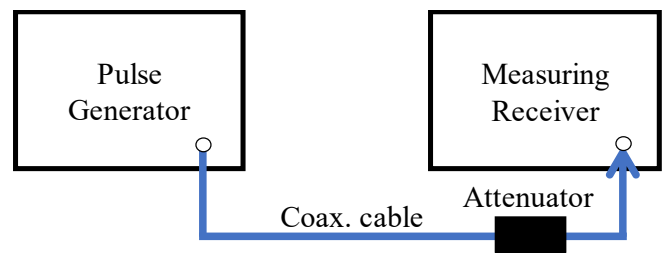

Fig. 1. Pulse response calibration method for EMI measuring receivers. 


\section{Calibration Pulse Standard Requirements}

The requirements given in CISPR 16-1-1 regarding the calibration pulses are limited to their amplitude level, frequency flatness, impulse area, and pulse repetition frequency.

On the one hand, the calibration pulses' spectrum must be flat in the frequency band under assessment (CISPR bands A, $\mathrm{B}$, and $\mathrm{C} / \mathrm{D}$ ) with a level equivalent to a tone having an RMS voltage of $2 \mathrm{mV}$, which means $66 \mathrm{~dB}(\mu \mathrm{V})$ at $50 \Omega$ source impedance and in open circuit conditions. In this regard, the spectrum of the pulses is considered sufficiently flat if the deviation of the spectrum amplitude is less than $\pm 2 \mathrm{~dB}$ relative to its value for the lower frequencies within the band. On the other hand, the spectrum above the upper limit of the frequency band shall be limited at least $10 \mathrm{~dB}$ down at twice the upper frequency.

Concerning the impulse area, $A_{\text {imp }}$, CISPR 16-1-1 give the specifications that are reproduced in Table I and indicates that it shall be known within $\pm 0.5 \mathrm{~dB}$ and the repetition frequency, $f_{\text {rep }}$, to within about $1 \%$. These flat pulses are applied for different repetition rates in order to obtain a certain ratio between peak, QP, AV, and RMS detectors, weighting the repetitiveness of the measured interferences [1].

TABLE I. STANDARD IMPULSE AREA SPECIFICATION

\begin{tabular}{ccc}
\hline \hline CISPR frequency band & $\boldsymbol{A}_{\text {imp }}[\mu \mathrm{Vs}]$ & $\boldsymbol{f}_{\text {rep }}{ }^{\mathrm{a}}[\mathrm{Hz}]$ \\
\hline \hline $\mathrm{A}(9 \mathrm{kHz}-150 \mathrm{kHz})$ & 13.5 & $1,2,5,10, \mathbf{2 5}, 60,100$ \\
$\mathrm{~B}(150 \mathrm{kHz}-30 \mathrm{MHz})$ & 0.316 & $1,2,10,20, \mathbf{1 0 0}, 1000$ \\
$\mathrm{C} / \mathrm{D}(30 \mathrm{MHz}-1 \mathrm{GHz})$ & 0.044 & $1,2,10,20, \mathbf{1 0 0}, 1000$ \\
\hline
\end{tabular}

${ }^{a}$ The reference repetition frequency for relative calibration for each CISPR band is highlighted in bold.

Finally, the standard recognizes that above $1 \mathrm{GHz}$ it is not feasible to generate pulses with the amplitudes and the rise time that would be required. Consequently, CISPR 16-1-1 indicates that: "Above $1 \mathrm{GHz}$, the required impulse area is defined using a pulse-modulated carrier at the frequency of test...". Previously, alternative methods have been proposed for this purpose [2].

\section{WAVEFORM CHARACTERIZATION}

During an intercomparison exercise held as an activity of the "Development of RF and Microwave Metrology Capability," (RFMicrowave, 15RPT01) project, an EMI Calibration Pulse Generator type IGUU 2916 manufactured by Schwarzbeck Mess-Elektronik (S/N 164) and provided by TÜBİTAK UME was used as a traveling standard [8].

Particularly, the focus of this intercomparison exercise was to measure the spectrum of the pulses. Nonetheless, complementary waveform measurements were performed using the setup shown in Fig. 1 but replacing the measuring receiver with a digital real-time sampling oscilloscope (DRTO). Table II includes the pulse characteristics calculated from the beforementioned measurements.

Figures 2 to 4 show the IGUU 2916 waveforms measured with the oscilloscope DPO5014B from Tektronix after applying the correction factors for compensating the signal path attenuation (cable and a $20 \mathrm{~dB}$ external attenuator). Equivalent-time sampling was used at $400 \mathrm{GSa} / \mathrm{s}$ with a bandwidth of $1 \mathrm{GHz}$. $A_{\text {imp }}$ was equal to $13.38 \mu \mathrm{Vs}, 0.306 \mu \mathrm{Vs}$, and $0.0446 \mu \mathrm{Vs}$ for bands $\mathrm{A}, \mathrm{B}$ and $\mathrm{C} / \mathrm{D}$ respectively, which is satisfactory in terms of the standard specifications.
TABLE II. PULSE CHARACTERISTICS OF THE IGUU 2916 WAVEFORMS

\begin{tabular}{r|c|c|c}
\multicolumn{2}{c}{ Characteristic } & \multicolumn{3}{|c}{ CISPR Band } \\
\cline { 2 - 4 } & A & B & C/D \\
\hline Rise time, $t_{r, 10-90 \%}[\mathrm{ps}]$ & 472.40 & 464.81 & 370.32 \\
\hline Fall time, $t_{f, 90-10 \%}[\mathrm{~ns}]$ & 28.67 & 0.39 & 0.24 \\
\hline Pulse width, $\tau[\mathrm{ns}]$ & 263.91 & 6.14 & 4.46 \\
\hline Mean voltage of the upper state, $U_{\operatorname{high}}[\mathrm{V}]$ & 50.47 & 51.36 & 107.17 \\
\hline Max. level, $U_{\max }[\mathrm{V}]$ & 52.14 & 51.93 & 107.20 \\
\hline $0 \mathrm{~dB} /$ decade breakpoint $=1 /(\pi \tau)[\mathrm{MHz}]$ & 1.21 & 51.85 & 713.19 \\
\hline $20 \mathrm{~dB} /$ decade breakpoint $=1 /\left(\pi t_{r}\right)[\mathrm{MHz}]$ & 673.81 & 684.82 & 859.56 \\
\hline Bandwidth, $\mathrm{BW}=1 / t_{r}[\mathrm{GHz}]$ & 2.11 & 2.15 & 2.7 \\
\hline \hline
\end{tabular}

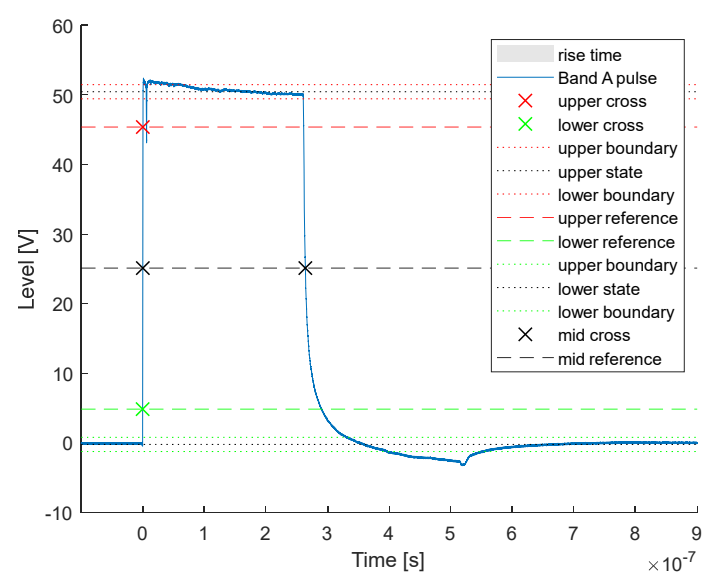

Fig. 2. Calibration pulse generated by the IGUU 2916 for CISPR band A.

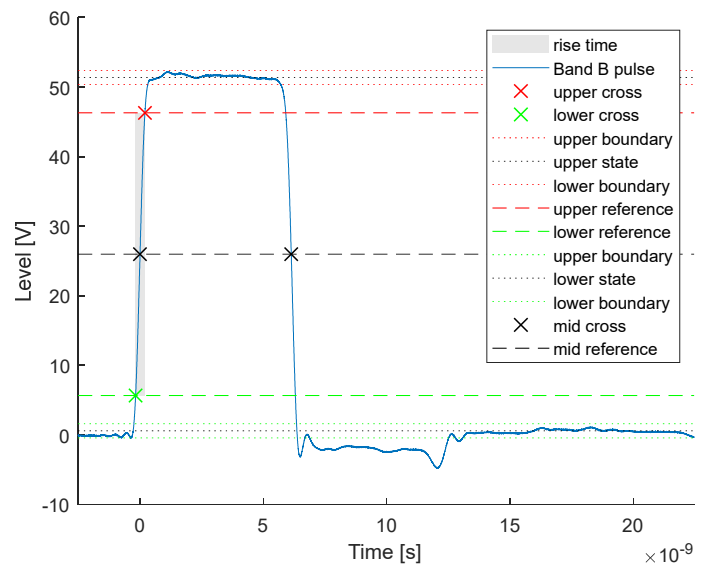

Fig. 3. Calibration pulse generated by the IGUU 2916 for CISPR band B.

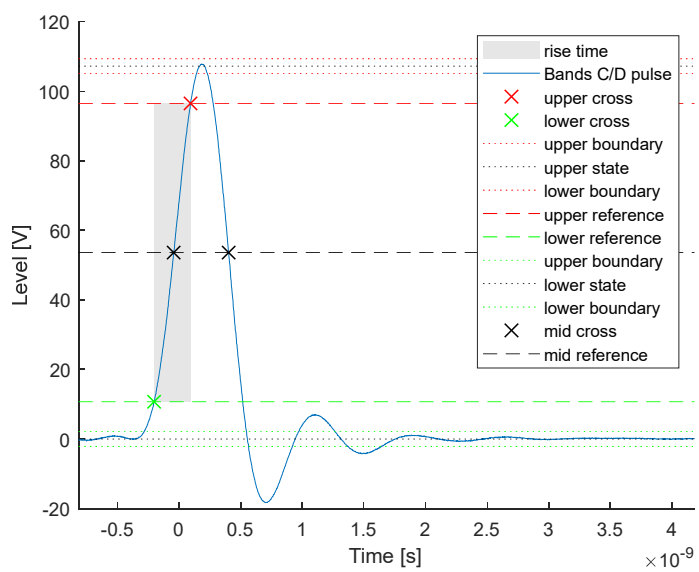

Fig. 4. Calibration pulse generated by the IGUU 2916 for CISPR bands C/D 
From the results above, it is important to notice that:

- In all cases, the measured rise time was relatively similar, in the sub-nanosecond range.

- The rise and fall times were not necessarily similar.

- The maximum voltage levels of the calibration pulses are potentially destructive for the inputs of some measuring receivers. In fact, this is warned by the pulse generator manufacturer in its manual.

- The bandwidth of the baseband pulses exceeds the frequency range of the corresponding CISPR bands. For CISPR bands A and B the $0 \mathrm{~dB} /$ decade decay frequency breakpoint is above the maximum frequency of the band. This means the calibration pulses have a significant amount of energy spread in frequencies above the frequency band under assessment.

- If the measured waveforms are considered as approximately rectangular, their impulse area could be estimated as the pulse width times the mean voltage of the upper state, that is, $A_{\text {imp }}=\tau \times U_{\text {high }}$. Accordingly, the difference between the impulse area calculated using the rectangular approximation and the direct integration of the measured waveform area is $0.06 \mathrm{~dB}, 0.17 \mathrm{~dB}$ and $0.66 \mathrm{~dB}$ for CISPR bands $\mathrm{A}, \mathrm{B}$, and $\mathrm{C} / \mathrm{D}$, respectively.

Regarding the measurements above, one could argue that it would be advisable to have used an oscilloscope of higher bandwidth for characterizing such calibration pulses given their short duration and high slew rate. That being said, it is important to acknowledge this limitation of the DRTO, and the forthcoming analysis will be restricted to CISPR bands A and $\mathrm{B}$. In fact, bands $\mathrm{A}$ and $\mathrm{B}$ are the ones in which using such baseband pulses is reasonable, while for higher frequencies, the modulated pulse approach is a much more suitable technique.

Alternatively, for bands $\mathrm{C} / \mathrm{D}$, baseband calibration pulses have been reported to be measured using the setup in Fig. 5 . There, a high bandwidth (i.e. $6 \mathrm{GHz}$ ) equivalent-time sampling oscilloscope (DSO) is employed for capturing the waveforms and the trigger signal is derived from the measured signal itself through a power divider and a delay line. This calibration method can be made traceable to the electro-optic sampling system [9]. Deconvolution of the measurement system from the measured signal is discussed in detail here [10].

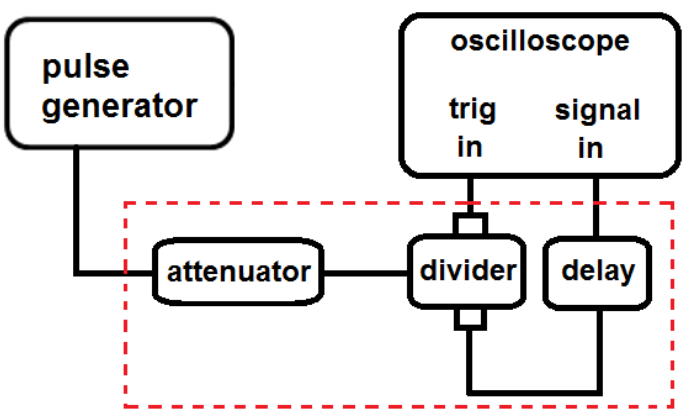

Fig. 5. Typical measurement setup for the calibration of the pulse generators using equivalent-time sampling oscilloscope.

\section{From THE WAVEFORM TO THE SPECTRUM}

One option for calculating the spectrum of the calibration pulses is to compute the Fourier transform of the time-domain waveform. In that sense, applying the Fast Fourier Transform (FFT) to the recorded voltage waveform would be enough for obtaining a numeric result. However, the oscilloscope epoch is limited by the memory depth and, therefore, the number of samples might be insufficient for obtaining a good FFT resolution.

It is important to keep in mind that if the highest time resolution is employed in order to measure pulse area with the maximum possible density of waveform points, then the resulting frequency resolution is minimum. For instance, a DRTO with a memory depth of $50 \mathrm{MSa}$ that is sampling the waveform at $400 \mathrm{GSa} / \mathrm{s}$ would have an FFT resolution of $8 \mathrm{kHz} / \mathrm{bin}$. This is a too coarse frequency resolution for CISPR band $\mathrm{A}$ and $\mathrm{B}$ if we think the minimum recommendable step size is half the resolution bandwidth, in other words, $100 \mathrm{~Hz}$ and $4.5 \mathrm{kHz}$, respectively. Similarly, if the record length covers a shorter time interval around the pulse, the achievable frequency resolution might be even worse.

One option would be windowing the acquired data and then use zero padding before the FFT calculations in order to achieve the required resolution. Then, Fourier transform of the voltage trace from oscilloscope in [V], $V(f)$, should be transformed into the spectral density, $S(f)$, accordingly,

$$
S(f)=20 \log \left(\frac{|V(f)| \sqrt{2} \cdot 10^{6} \cdot k_{A T T} \cdot k_{o s c}}{N_{F F T} \Delta f / 10^{6}}\right) \quad[\mathrm{dB} \mu \mathrm{V} / \mathrm{MHz}],
$$

where $N_{F F T}$ is the FFT length, $\Delta f$ is the frequency resolution in $[\mathrm{Hz}], k_{A T T}$ is the total attenuation of the signal path, that is, the cables and external attenuators connected between the generator and oscilloscope, $k_{\text {osc }}$ is a factor considering the oscilloscope frequency response.

However, at frequencies where $f \ll 1 / \tau$, the relationship (2) applies,

$$
S=20 \log \left(\sqrt{2} A_{\text {imp }}\right)+K[\mathrm{~dB} \mu \mathrm{V} / \mathrm{MHz}],
$$

where the impulse area, $A_{\text {imp }}$, is in $[\mu \mathrm{Vs}]$ and $K$ is the total correction factor in $[\mathrm{dB}]$. As the conditions are applicable for the calibration pulses above, using (2) is convenient because it provides a scalar relationship for the value of the spectrum density where the measurement uncertainty of the waveform area can be directly propagated to the spectral density.

Finally, since we are interested in comparing the measured pulse spectrum with the standard requirements, stated in terms of the QP level, it is necessary to perform a conversion that accounts for the impulse bandwidth and the peak to quasi-peak weighting ratio, that is,

$$
S_{Q P}=20 \log \left(\sqrt{2} A_{i m p} B_{i m p}\right)+K+W_{P K-Q P}[\mathrm{~dB} \mu \mathrm{V}]
$$

where $B_{i m p}$ is the impulse bandwidth in $[\mathrm{Hz}]$ and $W_{P K-Q P}$ is peak to quasi-peak weighting ratio at the reference pulse repetition frequency. If (3) is evaluated for CISPR bands A, $\mathrm{B}$ and $\mathrm{C} / \mathrm{D}$, the expression can be simplified as, 


$$
\begin{aligned}
& S_{Q P, A}=20 \log \left(A_{\text {imp }}\right)+K+43.39 \mathrm{~dB} \quad[\mathrm{~dB} \mu \mathrm{V}] \\
& S_{Q P, B}=20 \log \left(A_{\text {imp }}\right)+K+76.00 \mathrm{~dB}[\mathrm{~dB} \mu \mathrm{V}] \\
& S_{Q P, C D}=20 \log \left(A_{i m p}\right)+K+93.13 \mathrm{~dB} \quad[\mathrm{~dB} \mu \mathrm{V}]
\end{aligned}
$$

The formulas in (4), (5), and (6) can easily be verified by evaluating the quasi-peak amplitude spectrum of a pulse having the standard area while no correction factor is applied. The main limitation of the aforesaid expression is that it does not allow for a frequency dependent correction factor. Luckily, provided low loss cables and quality attenuators with flat frequency response, the attenuation factor can be assumed as constant and approximated to the mean attenuation value in the assessed band.

Regarding the impulse area, $A_{\text {imp }}$, this is obtained by integrating the calibration pulse waveform, $p(t)$, over one period, $T$. Since the measured pulse waveform is a timediscrete amplitude-discrete signal, the impulse area can be approximated by numerical integration using the trapezoidal rule [11], which means,

$$
A_{\text {imp }}=\int_{0}^{T} p(t) d t \approx A_{\text {imp }}{ }^{\prime}=\sum_{n=1}^{N} \frac{p\left[t_{n-1}\right]+p\left[t_{n}\right]}{2} \Delta t
$$

where $\Delta t$ is the sampling period, $N$ is the number of samples and $n$ is the discrete time index. Then, the numerical integration error, $e_{A}$, can be estimated using Richardson's extrapolation formula [11], that is,

$$
e_{A} \approx \frac{1}{2^{q}-1}\left[A_{i m p}{ }^{\prime}\left(f_{s}\right)-A_{i m p}{ }^{\prime}\left(\frac{f_{s}}{2}\right)\right]
$$

where $p$ is called the order of convergence, and $2^{q}$ is the convergence ratio given by,

$$
2^{q} \approx \frac{A_{\text {imp }}{ }^{\prime}\left(\frac{f_{s}}{2}\right)-A_{\text {imp }}{ }^{\prime}\left(\frac{f_{s}}{4}\right)}{A_{\text {imp }}{ }^{\prime}\left(f_{s}\right)-A_{\text {imp }}{ }^{\prime}\left(\frac{f_{s}}{2}\right)}
$$

It is important to note that, to reduce the error in the impulse area due to the noise in the measured waveform (thermal, quantization, and residual noise from the generator) it is advisable to low-pass filter $p(t)$. In such a case, the cutoff frequency must be set to be at least twice the upper limit of the frequency band corresponding to the pulse waveform. Other waveform noise reduction techniques such as high-resolution acquisition modes (box-car filter), waveform averaging and setting appropriate hardware filters in the oscilloscope prior the acquisition also contribute to reducing the variability of the measured impulse area [12].

Table III shows the measured impulse area, the estimated numerical integration error, the corresponding correction factor, the resulting quasi-peak spectrum level, and, finally, the deviation from the standard requirement. The numeric integration error is negligible at the sampling rate set. The observed deviation is within the tolerance interval of $\pm 2 \mathrm{~dB}$ defined by the CISPR 16-1-1 standard.
TABLE III. MEASURED QuASI-PEAK SPECTRUM LEVEL FOR THE CAlibration Pulses

\begin{tabular}{cccccc}
\hline \hline $\begin{array}{c}\text { CISPR } \\
\text { frequency } \\
\text { band }\end{array}$ & $\begin{array}{c}\boldsymbol{A}_{\text {imp }} \\
{[\mu \mathrm{Vs}]}\end{array}$ & $\begin{array}{c}\boldsymbol{e}_{\boldsymbol{A}} \\
{[\mu \mathrm{Vs}]}\end{array}$ & $\begin{array}{c}K \\
{[\mathrm{~dB}]}\end{array}$ & $\begin{array}{c}\boldsymbol{S}_{\boldsymbol{Q P}} \\
{[\mathrm{dB} \mu \mathrm{V}]}\end{array}$ & $\begin{array}{c}\text { Deviation } \\
{[\mathrm{dB}]}\end{array}$ \\
\hline \hline $\mathrm{A}$ & 1.3304 & $-9 \mathrm{e}-9$ & 20.05 & 65.92 & 0.08 \\
$\mathrm{~B}$ & 0.03020 & $-3.4 \mathrm{e}-8$ & 20.13 & 65.73 & 0.27 \\
$\mathrm{C} / \mathrm{D}$ & 0.00427 & $-6.8 \mathrm{e}-9$ & 20.38 & 66.12 & -0.12 \\
\hline
\end{tabular}

\section{MEASUREMENT UNCERTAINTY}

Estimating the measurement uncertainty in the quasi-peak spectrum amplitude level requires adding contributions from three mains sources of uncertainty, namely, the correction factors, the impedance mismatch, and the impulse area. Each of them includes other underlying sources of uncertainty that are broken down in the measurement uncertainty budgets shown in Tables IV to VI. The budget is based on the method of the Guide to the Expression of Uncertainty in Measurement and its recommended application for EMC testing [13], [14].

The measurement uncertainty was calculated for each CISPR band separately, even if the measurement method and setup remain the same for the calibration of the pulse generator in all the CISPR bands under consideration. The reason for this approach is to adjust the estimated measurement uncertainty according to the frequency.

Firstly, the uncertainty of the correction factors is compound by the contributions of the cable-attenuator attenuation correction, $u_{A T T}$, and the correction due to the oscilloscope transfer function, $u_{O S C}$.

On the one hand, the attenuation of the signal path is measured using a Vector Network Analyzer. $S_{Q P}$ is corrected with the mean attenuation value in the corresponding frequency range. Since the attenuation is frequency dependent, $u_{A T T}$ is estimated as the standard deviation of the attenuation in the whole frequency band.

Conversely, no correction is applied due to the oscilloscope transfer function. In this regard, $u_{O S C}$ is estimated based on manufacturer specifications and assuming the oscilloscope frequency response corresponds to a Gaussian filter with a $3 \mathrm{~dB}$ bandwidth of $1 \mathrm{GHz}$. This is reasonable for most DRTO because the one pole roll-off characteristic response in oscilloscopes helps to reduce the overshoot and the ringing in digital waveforms and allows for more accurate rise time measurements. However, oscilloscopes might have another type of response such as Maximally flat, Chebyshev, or Butterworth and this affects waveform fidelity [15].

Secondly, the uncertainty due to impedance mismatch between devices " 1 " and " 2 " and expressed in decibels, $u_{M}$, is calculated as follows,

$$
u_{M}=20 \log \left(1+\left|\Gamma_{1}\right|\left|\Gamma_{2}\right|\right)
$$

where $\Gamma_{1}$ and $\Gamma_{2}$ are the reflection coefficients of devices 1 and 2 , respectively. For the calibration setup, this uncertainty contribution must be accounted not only for the generatorattenuator mismatch but also for the attenuator-oscilloscope mismatch. 
Thirdly, the uncertainty in the measurement of the impulse area, $u_{\text {Area }}$, is estimated as the standard deviation of the mean value of the impulse area calculated for independently acquired waveforms of different occurrences of the generated pulse. The mean value of the impulse area, $\bar{A}_{i m p}$, is computed over several repetitions of $A^{\prime}{ }_{i m p}$ for a single pulse repetition frequency. Usually, the number of waveform acquisitions, $n$, is larger than 30 . This uncertainty is evaluated as a Type A contribution, that is to say,

$$
u_{\text {Area }}=\sqrt{\frac{1}{n(n-1)} \sum_{i=1}^{n}\left(A_{i m p}^{\prime}-\bar{A}_{i m p}\right)^{2}} .
$$

For each setting of the pulse repetition frequency and amplitude, the waveforms of the pulses have good repeatability. Area variations are slight and can be related to the noise in the time-domain voltage trace. When appropriate filtering is applied, and the waveform is averaged over a significant number of repetitions, it is possible to reduce $u_{\text {Area }}$ to about $0.1 \%$. However, it is important to acknowledge the pulse generator waveforms, and thus the impulse area, change with the repetition frequency. Finally, the uncertainty due to numerical integration errors has been considered as negligible provided a sufficiently high sampling frequency.

\begin{tabular}{|c|c|c|c|c|c|}
\hline \multicolumn{6}{|c|}{$\begin{array}{l}\text { TABLE IV. UNCERTAINTY BUDGET FOR THE CALIBRATION-PULSE } \\
\text { QUASI-PEAK SPECTRUM AMPLITUDE LEVEL. BAND A }\end{array}$} \\
\hline & $\begin{array}{c}\text { Estimate } \\
\text { uncertainty } \\
{[\mathrm{dB}]}\end{array}$ & $\begin{array}{l}\text { Probability } \\
\text { distribution }\end{array}$ & Divisor & $\begin{array}{c}\text { Standard } \\
\text { uncertainty } \\
{[\mathrm{dB}]} \\
\end{array}$ & $\begin{array}{c}\text { Uncertainty } \\
\text { contribution } \\
{[\mathrm{dB}]}\end{array}$ \\
\hline$u_{A t t}$ & 0.02 & Normal & 1 & 0.020 & 0.020 \\
\hline uosC & 0.06 & Normal & 1 & 0.060 & 0.060 \\
\hline$u_{M(A T T-o s c)}$ & 0.008 & U-shaped & 1.41 & 0.006 & 0.006 \\
\hline$u_{M(g e n-A T T)}$ & 0.001 & U-shaped & 1.41 & 0.001 & 0.001 \\
\hline$u_{\text {Area }}$ & 0.01 & Normal & 1 & 0.010 & 0.010 \\
\hline \multicolumn{5}{|c|}{ Combined standard uncertainty } & 0.064 \\
\hline \multicolumn{5}{|c|}{ Expanded uncertainty $(k=2)$} & $\pm 0.13 \mathrm{~dB}$ \\
\hline
\end{tabular}

\begin{tabular}{|c|c|c|c|c|c|}
\hline & $\begin{array}{c}\text { Estimate } \\
\text { uncertainty } \\
{[\mathrm{dB}]}\end{array}$ & $\begin{array}{l}\text { Probability } \\
\text { distribution }\end{array}$ & Divisor & $\begin{array}{c}\text { Standard } \\
\text { uncertainty } \\
{[\mathrm{dB}]}\end{array}$ & $\begin{array}{c}\text { Uncertainty } \\
\text { contribution } \\
{[\mathrm{dB}]}\end{array}$ \\
\hline$u_{\text {Att }}$ & 0.040 & Normal & 1 & 0.040 & 0.020 \\
\hline$u_{O S C}$ & 0.070 & Normal & 1 & 0.070 & 0.060 \\
\hline$u_{M(A T T-o s c)}$ & 0.006 & U-shaped & 1.41 & 0.006 & 0.006 \\
\hline$u_{M(g e n-A T T)}$ & 0.001 & U-shaped & 1.41 & 0.001 & 0.001 \\
\hline$u_{\text {Area }}$ & 0.020 & Normal & 1 & 0.020 & 0.010 \\
\hline \multicolumn{5}{|c|}{ Combined standard uncertainty } & 0.083 \\
\hline \multicolumn{5}{|c|}{ Expanded uncertainty $(k=2)$} & $\pm 0.17 \mathrm{~dB}$ \\
\hline
\end{tabular}

TABLE VI. UNCERTAINTY BUDGET FOR THE CALIBRATION-PULSE

\begin{tabular}{|c|c|c|c|c|c|}
\hline & $\begin{array}{c}\text { Estimate } \\
\text { uncertainty } \\
{[\mathrm{dB}]}\end{array}$ & $\begin{array}{l}\text { Probability } \\
\text { distribution }\end{array}$ & Divisor & $\begin{array}{c}\text { Standard } \\
\text { uncertainty } \\
{[\mathrm{dB}]}\end{array}$ & $\begin{array}{c}\text { Uncertainty } \\
\text { contribution } \\
{[\mathrm{dB}]}\end{array}$ \\
\hline$u_{A t t}$ & 0.06 & Normal & 1 & 0.060 & 0.060 \\
\hline UOSC & 0.1 & Normal & 1 & 0.1 & 0.1 \\
\hline$u_{M(A T T-o s c)}$ & 0.03 & U-shaped & 1,41 & 0.021 & 0.021 \\
\hline$u_{M(g e n-A T T)}$ & 0.005 & U-shaped & 1,41 & 0.004 & 0.004 \\
\hline$u_{\text {Area }}$ & 0.05 & Normal & 1 & 0.05 & 0.05 \\
\hline \multicolumn{5}{|c|}{ Combined standard uncertainty } & 0.129 \\
\hline \multicolumn{5}{|c|}{ Expanded uncertainty $(k=2)$} & $\pm 0.26 \mathrm{~dB}$ \\
\hline
\end{tabular}

\section{CONCLUSIONS}

The waveform characterization of the calibration pulse generators provides a comprehensive approach for determining the fundamental parameters that define the reference waveforms and it allows verifying compliance in terms of the CISPR 16-1-1 requirements for the pulse area and quasi-peak amplitude level. Previously, DRTO and DSO have been successfully used for calibrating the pulse generators with a focus on obtaining the spectrum density through the Fourier transform. However, in this case, we have prioritized the impulse area measurements and then calculated the spectrum density using the approximation in (2). Consequently, the waveforms were sampled at the maximum sampling rates for minimizing the uncertainty due to numerical integration. The measurement uncertainty is within the target $\pm 0.5 \mathrm{~dB}$ given in standards for CISPR band $\mathrm{A}, \mathrm{B}$ and $\mathrm{C} / \mathrm{D}$. According to the results, the pulse generator IGUU 2916 is confirmed to meet the expected performance for a confidence level larger than $95 \%$.

\section{ACKNOWLEDGMENT}

This work was supported by the project 15RPT01 RFMicrowave. This project has received funding from the EMPIR programme co-financed by the Participating States and from the European Union's Horizon 2020 research and innovation programme.

\section{REFERENCES}

[1] IEC CISPR, 16-1-1 ed4.0: Specification for radio disturbance and immunity measuring apparatus and methods - Part 1-1: Radio disturbance and immunity measuring apparatus - Measuring apparatus. IEC, 2015.

[2] M. A. Azpúrua et al., "Waveform Approach for Assessing Conformity of CISPR 16-1-1 Measuring Receivers," IEEE Trans. Instrum. Meas., vol. 67, no. 5, pp. 1187-1198, 2018.

[3] M. A. Azpúrua, M. Pous, J. A. Oliva, and F. Silva, "Fast and automated verification of multi-channel full time-domain EMI measurement system," in 2017 IEEE International Instrumentation and Measurement Technology Conference (I2MTC), 2017, pp. 1-6.

[4] I. Agilent Technologies, Calibration Guide. 8590L Spectrum Analyzer. USA, 2001.

[5] I. Agilent Technologies, Calibration Guide. Agilent Technologies EMC Analyzers. USA, 2012.

[6] Fluke, "A Guide to Calibrating Your Spectrum Analyzer," 2006.

[7] R. D. Drosd, "Transmission line pulse generator," 1956.

[8] "Publishable Summary for 15RPT01 RFMicrowave Development of RF and microwave metrology capability," 2015.

[9] M. Bieler et al., "Rise-time calibration of $50-\mathrm{GHz}$ sampling oscilloscopes: Intercomparison between PTB and NPL," in IEEE Transactions on Instrumentation and Measurement, 2007.

[10] P. D. Hale et al., "Traceable waveform calibration with a covariancebased uncertainty analysis," IEEE Trans. Instrum. Meas., 2009.

[11] D. W. Arthur, P. J. Davis, and P. Rabinowitz, "Methods of Numerical Integration," Math. Gaz., 2007.

[12] M. A. Azpúrua, M. Pous, S. Çakir, M. Çetinta, and F. Silva, "Improving time-domain EMI measurements through digital signal processing," IEEE Electromagn. Compat. Mag., vol. 4, no. 2, pp. 82-91, 2015.

[13] Joint Committee For Guides In, "Evaluation of measurement data Guide to the expression of uncertainty in measurement," Int. Organ. Stand. Geneva ISBN, 2008.

[14] M. A. Azpúrua, C. Tremola, and E. Paez Barrios, "Comparison of the Gum and Monte Carlo Methods for the Uncertainty Estimation in Electromagnetic Compatibility Testing," Prog. Electromagn. Res. B, vol. 34, no. September, pp. 125-144, 2011.

[15] H. W. Johnson, High-Speed Digital Design: A Handbook of Black Magic. 1993. 
